\title{
Alveolar Ridge Preservation Using Grafting Material in Molar Region
}

ISSN: 2637-7764

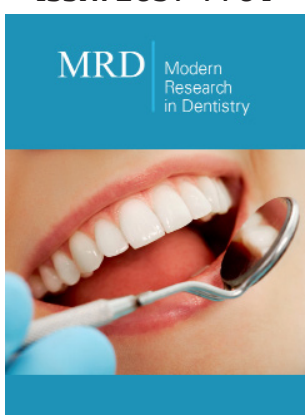

*Corresponding author: Shaoyun Jiang, Professor, Stomatological Center, Peking University Shenzhen Hospital, Guangdong Provincial High-level Clinical Key Specialty, Guangdong Province Engineering Research Center of Oral Disease Diagnosis and Treatment, Shenzhen, 518036, P.R. China

Submission: 制April 21, 2020

Published: 㘹May 06, 2021

Volume 6 - Issue 3

How to cite this article: Mengnan Shi, Shaoyun Jiang. Alveolar Ridge Preservation Using Grafting Material in Molar Region. Mod Res Dent. 6(3). MRD. 000637. 2021. DOI:_10.31031/MRD.2021.06.000637

Copyright@ Shaoyun Jiang, This article is distributed under the terms of the Creative Commons Attribution 4.0 International License, which permits unrestricted use and redistribution provided that the original author and source are credited.

\author{
Mengnan Shi ${ }^{1}$ and Shaoyun Jiang ${ }^{2 *}$
}

${ }^{1}$ Dentist, Department of Stomatology, Southern University of Science and Technology Hospital, Shenzhen, Guangdong, P. R. China; Postgraduate Candidate, School of Dentistry, Tianjin Medical University, Tianjin, P. R. China

${ }^{2}$ Professor, Stomatological Center, Peking University Shenzhen Hospital, Guangdong Provincial High-level Clinical Key Specialty, Guangdong Province Engineering Research Center of Oral Disease Diagnosis and Treatment, Shenzhen, P. R. China

\begin{abstract}
It should be considered how to maintain the soft and hard tissues during tooth extraction in order to improve post-restoration. Thus, Alveolar ridge preservation (ARP) has been proposed. Grafting materials have been being used to preserve the alveolar ridge in molar area. Based on the special structure of molar area, the effect of ARP using grafting materials in molar area and the related-influence factors will be discussed in this review.
\end{abstract}

Keywords: Alveolar ridge preservation; Molar; Grafting materials

\section{Introduction}

After tooth extraction, alveolar ridge exhibits three-dimension remodeling during natural healing, which shows the buccolingual reduction in bone thickness (average $-3.87 \mathrm{~mm}, 95 \% \mathrm{CI}$ : -4.059 to $-3.673 \mathrm{~mm}$ ) in the ridge, and vertical mid-buccal resorption in bone height (average $-1.67 \mathrm{~mm}, 95 \%$ CI: -1.910 to $-1.428 \mathrm{~mm}$ ) [1]. To solve this problem, alveolar ridge preservation (ARP) was proposed to block the resorption of alveolar bone and realize the preservation or increment of alveolar bone [2]. Except for immediate implant, ARP using grafting materials has been applied in tooth extraction. It has shown that ARP prevents $1.5-2.4 \mathrm{~mm}$ in horizontal, $1.0-2.5 \mathrm{~mm}$ in vertical mid-buccal, and $0.8-1.5 \mathrm{~mm}$ in mid-lingual vertical bone resorption [3]. Although multi-root teeth were distinguished from single ones in this study, premolars and molars were taken as multi-root teeth. Considering the peculiar structure of molars such as the larger extraction sockets, the greater angle of root bifurcation, the bone resorption induced by periodontitis, the effect of ARP using grafting materials in molar area may be different. This review summarizes the effect of ARP using grafting materials in the molar area and the related-influence factors, based on the current clinic studies of ARP using grafting materials in molar area from 2010 to 2021.

\section{The effect of ARP using grafting materials in molar area}

Bone grafts: In ARP, bone grafts mainly lead to osteoinduction and/or osteoconduction [4]. Currently, there are mainly three categories including allograft, xenograft, and alloplastic graft in ARP in molar area.

As known, allograft bone is effective bone regeneration material. Walker et al applied the allogeneic mineralized cortical freeze-dried bone allograft (FDBA) covered with dense Polytetrafluoroethylene (d-PTFE) in molar extraction sites in a randomized clinical trial (RCT), observed that the loss of ridge height in the test group was significantly less than that in the control group (natural healing) $(1.12 \mathrm{~mm}$ and $2.60 \mathrm{~mm}$, respectively) after 3 months, and that sites needing bone grafting were $10 \%$ in the test group versus $25 \%$ in the control group in further implant [5]. Similarly, Harthi et al. [6] performed ARP with FDBA and CollaPlug (sponge type; native collagen), found that the test group was significantly less loss of bone height. However, both studies did not show the statistical intergroup difference of the change of alveolar ridge width. 
Demineralized bovine bone matrix (DBBM) has been widely applied in the regeneration of the alveolar bone. Kim et al. [7] evaluated the effect of DBBM with Teruplug (sponge type; crosslinked collagen) after molar extraction in RCT and found that the resorption of alveolar bone width in the test group was significantly less than that in the control group $(14.26 \%$ and $20.74 \%$, respectively) after 3 months. Lim et al. [8] obtained similar results when using DBBM with Bio-Gide (film type; natural collagen) in molar extraction sites [8]. Even when DBBM with BioGide was applied in periodontally compromised sites, bone width and buccal bone height increased in the test group while decreased in the control group [9]. The three studies supported an increasing trend in bone width after ARP using grafting materials in molar area. However, it is still controversial. One research showed that the vertical height of alveolar bone, rather than horizontal width, significantly increased after ARP using DBBM with Bio-Gide in molar area [10].

Alloplastic grafts have been used increasingly in bone engineering. Lombardi et al. [11] applied polylactic acidhydroxyacetic acid copolymer as a bone graft covered with Hemocollagene (sponge type; native collagen) in the extraction sites of maxillary first molars. Although there was no statistical difference in the change of bone volume between intergroup comparisons after 6 months, ARP decreased sinus pneumatization [11]. In mandibular molar sites, a novel bone grafting material composed of $\beta$-tricalcium phosphate and calcium sulfate with Jason (sponge type; native collagen) was applied to preserve sufficient bone, in which implant loading stability was maintained after 2 years of implantation [12]. However, the clinical benefit of alloplastic grafts in ARP is still less than that of allograft or xenograft [3].

Barrier membrane: Barrier membrane, including nonresorbable (such as d-PTFE) and absorbable (such as collagen) membrane, assists tissue regeneration and serves as a physical barrier for bone grafts [4]. Collagen is categorized into natural collagen membranes and cross-linked collagen membranes, showing no significant differences in bone regeneration [13]. The products of collagen membranes have sponge type and thinfilm type. The sponge type (e.g. Collaplug, Collatape, Teruplug, Hemocollagene, Jason fleece) fully embodies the advantages of collagen materials (biocompatibility, biodegradability, high tensile strength of three-dimensional scaffolds). The thin-film type not only has the biological advantages of collagen but also can be used as a physical barrier. For example, Bio-Gide (bilayer membrane) can guide the regeneration of soft tissue and act as a barrier to prevent the fibrous tissue from entering the space of bone graft [13]. Duong et al compared the effect of d-PTFE and Collaplug on ARP using FDBA and found that Collaplug was more beneficial to bone formation after 3 months [14], which needs to be further confirmed.

Growth factors: Growth factors can facilitate bone formation and/or vascularization [4]. $1.5 \mathrm{mg} / \mathrm{mL}$ recombinant human bone morphogenetic protein-2 (rhBMP2) in ARP effectively reduced bone width resorption [15]. Also, leucocyte- and platelet-rich fibrin
(L-PRF) decreased buccal bone resorption [16]. Autologous platelet concentrates (APCs, including platelet-rich plasma protein, growth factor-rich plasma protein, and platelet-rich fibrous plasma protein) may enhance the bone density and facilitates soft tissue healing, but did not contribute a significant increase of the percent of vital bone in ARP at molar extraction sites [17]. However, few clinical studies of other growth factors (e.g., growth factor concentrate, recombinant human fibroblast growth factor 2, growth differentiation factor 5) were applied in ARP, which needs to be further explored.

Above all, although there is still no evidence which material has the greatest advantage over others, it is definite that ARP using grafting materials in molar extraction area has certain benefit in reducing the resorption of ridge bone so as to reduce the need for bone augmentation surgery while implantation.

\section{Related-influence factors of ARP using grafting materials in molar area}

Periodontally compromised sites: At the extraction sites of periodontally compromised molars, DBBM with Bio-Gide increased the ridge width at $1 \mathrm{~mm}$ apical from the crest of alveolar bone and buccal ridge height, decreased the loss of lingual bone height compared with the control group ( -0.51 versus $-1.31 \mathrm{~mm})$ [9]. Also, the bone height of the central- and distal-buccal sites increased after using DBBM with Bio-Gide in the extraction sockets of molars with periodontitis, while bone width did not change [10]. According to this information, ARP using grafting materials in the extraction socket of periodontally compromised molars may be safe and effective in maintaining bone volume. However, ARP cannot be performed in all periodontally compromised sites, especially in the sockets with severe infection, severe bone defect, or defect involving maxillary sinus [18].

Barrier membrane: The application of a barrier membrane is beneficial to preserve bone volume. It has been shown that ARP with DBBM and Bio-Gide in molar area was beneficial to preserve the bone volume, showing that there was significantly less vertical reduction $(-0.25$ vs. $-1.15 \mathrm{~mm})$ in the midcrestal area, relatively less horizontal resorption $(-1.02 \mathrm{vs}-2.49 \mathrm{~mm})$ and relatively more percentage of bone formation compared with ARP with DBBM [8], which supports, to some extent, that barrier membranes may increase the stability of the bone grafts, promote bone formation, reduce bone resorption.

The size of bone particles: It is controversial whether the particle size of bone graft is dependent on the size of the bony defect, such as larger size particle for ARP [19]. After comparison between two bone grafts with different particle size in ARP at molar extraction sockets, the research team did not find the advantage of demineralized bone matrix (DBM) with large particle size (between 125 and $710 \mathrm{~mm}$ ) over DBM with small particle size (2 to $4 \mathrm{~mm}$ ) after 3 months [20].

The thickness of the buccal bone: It has been demonstrated that there is a positive correlation between buccal bone thickness before extraction and the change of ridge dimension after ARP, especially for the thinner buccal bone $(<1.0-1.5 \mathrm{~mm})$ [2]. However, 
Walker et al. [5] did not find this correlation in ARP at molar extraction sites while using FDBA and d-PTFE. Meanwhile, other studies on ARP in the molar area did not analyze the correlation $[7-11,20,21]$. Thus, whether the thickness of the buccal bone has an influence on the bone volume after ARP needs to be further explored.

Surgical operation: During a surgical operation, the influence factors include compressive forces on graft materials, primary closure of ARP, minimally invasive tooth extraction, and debridement of tooth sockets.

Cho et al. [21] investigated whether different compressive forces on DBBM affect the effect of ARP in an RCT study and found that there was no statistical difference in the change of ridge volume between intergroup ( $30 \mathrm{~N}$ vs. $5 \mathrm{~N}$ ) after 3 months. However, the histomorphometric analysis demonstrated that the percentage of new bone formation was significantly greater in the $30 \mathrm{~N}$ group [21], in which the big compressive force accelerated the new bone formation due to stimulatory effects on angiogenesis and the higher expression of genes related to cell proliferation [19].

Although the evidence showed that ARP with primary closure or without has little difference in the change of bone volume [22], some studies showed that the position of the mucogingival junction changed and the width of keratinized gingiva decreased after primary closure $[9,23]$. Zhao et al. [9] observed that the width of keratinized gingiva was reduced by $1.1 \mathrm{~mm}$ in the ARP group with primary closure and reduced by $0.7 \mathrm{~mm}$ in the group without primary closure. Meanwhile, another study showed that the width of keratinized gingiva was reduced by $1.6 \mathrm{~mm}$ in the ARP group with primary closure and reduced by $0.3 \mathrm{~mm}$ in the ARP group without primary closure which was covered with a collagen sponge [24]. Nevertheless, these two studies did not show the statistical difference in bone volume between two groups with or without primary closure. It was suggested that ARP covering the doublelayered membrane is better for sites with larger defects, the risk of delayed healing, or the difficulty in maintaining oral hygiene [25].

The surgical trauma of the tooth extraction process is one of the iatrogenic factors causing the reduction of soft and hard tissue at the tooth extraction site, so minimally invasive tooth extraction is advocated. Minimally invasive tooth extraction requires adequate preoperative evaluation to select the most appropriate tooth extraction mode and tooth extraction instruments, that is to say, some new instruments, such as periosteum and new tooth extraction system, are used to minimize the trauma and reduce the inflammatory response and traumatic bone resorption after tooth extraction [25]. Minimally invasive extractions were commonly used before ARP in molar area [5,6,8-12,21,23]. Minimally invasive tooth extraction does not mean absolutely no flap, especially for special sites such as the presence of big cracking and fenestration of alveolar bone, so as to avoid incomplete removal of infected tissue.

Debridement and saline irrigation in the tooth extraction socket with chronic inflammation could significantly reduce the bacterial load, especially Aggregatibacter actinomycetemcomitans and Porphyromonas gingivalis, so as to induce the decay of the inflammatory response and promote the regeneration of hard and soft tissue [26]. A lot of researchers have confirmed that there were no obvious postoperative complications after debridement during ARP in molar area, including in periodontally compromised sites $[5-12,20,21,23]$.

\section{Conclusion}

It is believed that ARP using grafting materials in molar area can reduce bone resorption. However, with the limitation in current technology and materials, it is still a great challenge to restore the original bone volume. The success of ARP in molar area is related to many factors such as appropriate cases, ideal biomaterials, and proper operative protocol.

\section{References}

1. Van der Weijden F, Dell'Acqua F, Slot DE (2009) Alveolar bone dimensional changes of post-extraction sockets in humans: a systematic review. J Clin Periodontol 36(12): 1048-1058.

2. Tonetti MS, Jung RE, Avila Ortiz G, Blanco J, Cosyn J, et al. (2019) Management of the extraction socket and timing of implant placement: consensus report and clinical recommendations of group 3 of the $\mathrm{XV}$ European Workshop in Periodontology. J Clin Periodontol 46(Suppl 21): 183-194.

3. Avila Ortiz G, Chambrone L, Vignoletti F (2019) Effect of alveolar ridge preservation interventions following tooth extraction: a systematic review and meta-analysis. J Clin Periodontol 46(Suppl 21): 195-223.

4. Moy PK, Aghaloo T (2019) Risk factors in bone augmentation procedures. Periodontol 2000 81(1): 76-90.

5. Walker CJ, Prihoda TJ, Mealey BL, Lasho DJ, Noujeim M, et al. (2017) Evaluation of healing at molar extraction sites with and without ridge preservation: a randomized controlled clinical trial. J Periodontol 88(3): 241-249.

6. Al Harthi SM, Prihoda TJ, Mealey BL, Lasho DJ, Noujeim M, et al. (2019) Healing at molar extraction sites using freeze-dried bone allograft and collagen wound dressing: case series and three-arm analyses. Int J Oral Maxillofac Implants 34(5): 1202-1212.

7. Kim YK, Yun PY, Lee HJ, Ahn JY, Kim SG (2011) Ridge preservation of the molar extraction socket using collagen sponge and xenogeneic bone grafts. Implant Dent 20(4): 267-272.

8. Lim HC, Shin HS, Cho IW, Koo KT, Park JC (2019) Ridge preservation in molar extraction sites with an open-healing approach: A randomized controlled clinical trial. J Clin Periodontol 46(11): 1144-1154.

9. Zhao L, Xu T, Hu W, Chung KH (2018) Preservation and augmentation of molar extraction sites affected by severe bone defect due to advanced periodontitis: a prospective clinical trial. Clin Implant Dent Relat Res 20(3): 333-344.

10.Zhan Y, Hu W, Zhen M, Xu T, Lu RF (2015) Radiographic evaluation of ridge preservation after molar tooth extraction: a controlled clinical trial. Beijing Da Xue Xue Bao Yi Xue Ban 47(1): 19-26.

11. Lombardi T, Bernardello F, Berton F, Porrelli D, Rapani A, et al. (2018) Efficacy of alveolar ridge preservation after maxillary molar extraction in reducing crestal bone resorption and sinus pneumatization: a multicenter prospective case-control study. Biomed Res Int 2018: 9352130.

12. Fairbairn P, Leventis M, Mangham C, Horowitz R (2018) Alveolar ridge preservation using a novel synthetic grafting material: a case with twoyear follow-up. Case Rep Dent 2018: 6412806. 
13. Sbricoli L, Guazzo R, Annunziata M, Gobbatao L, Bressan E, et al. (2020) Selection of collagen membranes for bone regeneration: a literature review. Materials 13(3): 786

14. Duong M, Mealey BL, Walker C, Al Harthi S, Prihoda TJ, et al. (2020) Evaluation of healing at molar extraction sites with and without ridge preservation: a three-arm histologic analysis. J Periodontol 91(1): 74-82

15. Moslemi N, Khoshkam V, Rafiei SC, Bahrami N, Aslroosta H (2018) Outcomes of alveolar ridge preservation with recombinant human bone morphogenetic protein-2: A systematic review. Implant Dent 27(3): 351-362.

16. Castro AB, Meschi N, Temmerman A, Pinto N, Lambrechts P, et al. (2017) Regenerative potential of leucocyte- and platelet-rich fibrin. Part B: sinus floor elevation, alveolar ridge preservation and implant therapy. A systematic review. J Clin Periodontol 44(2): 225-234.

17. Del Fabbro M, Bucchi C, Lolato A, Corbella S, Testori T, et al. (2017) Healing of postextraction sockets preserved with autologous platelet concentrates. A systematic review and meta-analysis. J Oral Maxillofac Surg 75(8): 1601-1615.

18. Lai SY, Hou JX (2020) Progress in the application of alveolar ridge preservation at extraction sites in non-periodontitis and periodontitis patients. Zhonghua Kou Qiang Yi Xue Za Zhi 55(4): 266-270.

19. Delgado Ruiz R, Romanos GE, Alexandre Gerhke S, Moreno GG, MatéSánchez JE et al. (2018) Biological effects of compressive forces exerted on particulate bone grafts during socket preservation: Animal study. Clin Oral Implants Res 29(7): 792-801.
20. Hoang TN, Mealey BL (2012) Histologic comparison of healing after ridge preservation using human demineralized bone matrix putty with one versus two different-sized bone particles. J Periodontol 83(2): 174181.

21. Cho IW, Park JC, Shin HS (2017) A comparison of different compressive forces on graft materials during alveolar ridge preservation. J Periodontal Implant Sci 47(1): 51-63.

22. Lee J, Lee JB, Koo KT, Seol YJ, Lee YM (2018) Flap management in alveolar ridge preservation: A systematic review and meta-analysis. Int J Oral Maxillofac Implants 33(3): 613-621.

23. Zhao L, Hu W, Xu T, Zhan YL, Wei YP, et al. (2019) Two procedures for ridge preservation of molar extraction sites affected by severe bone defect due to advanced periodontitis. Beijing Da Xue Xue Bao Yi Xue Ban 51(3): 579-585.

24. Choi HK, Cho HY, Lee SJ, Cho IW, Shin HS, et al. (2017) Alveolar ridge preservation with an open-healing approach using single-layer or double-layer coverage with collagen membranes. J Periodontal Implant Sci 47(6): 372-380.

25. Araújo M G, Silva C O, Misawa M, Sukekava F (2015) Alveolar socket healing: what can we learn. Periodontol 2000 68(1): 122-134.

26. Kim JJ, Ben Amara H, Schwarz F, Kim HY, Lee JW, et al. (2017) Is ridge preservation augmentation at periodontally compromised extraction sockets safe? A retrospective study. J Clin Periodontol 44(10): 10511058. 